\title{
Administración de hierro intravenoso en la consulta de nefrología
}

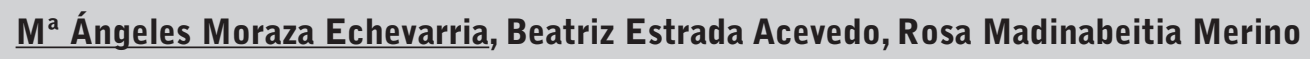

\section{Hospital Universitario de Álava. Txagorritxu. Álava}

\section{Introducción:}

El tratamiento de la anemia es una parte muy importante de los pacientes con enfermedad renal crónica. Las guías recomiendan: ferritina $>100 \mathrm{ng} / \mathrm{ml}$ e Indice de saturación $>20 \%$. Con el aporte de Fe oral es muy difícil alcanzar estos valores en muchos pacientes, por lo que se requiere de aporte de $\mathrm{Fe}$ intravenoso (i.v.) El hierro i.v. es una medicación de uso hospitalario y en muchos centros se pone en el hospital de día, pero actualmente están saturados con tratamientos oncológicos, por lo que es difícil encontrar hueco a nuestros pacientes. La administración de Fe i.v. por parte de la consulta de nefrología, nos permite en primer lugar adaptabilidad a la forma de pautar el hierro y una mayor comodidad para el paciente, aprovechando la extracción de sangre para los análisis con la misma punción venosa. También, al ser enfermeras especialistas en diálisis tenemos especial cuidado en cuidar el capital venoso de los pacientes para permitir en el futuro la realización de un acceso vascular. Todo ello se enmarca dentro del concepto de "hospital de día de nefrología" donde la enfermera no solo realiza actividades propias de la consulta sino que también hace actividades específicas de la especialidad de nefrología como cuidado del acceso vascular, consejo dietético e información sobre los tipos de tratamiento renal sustitutivo. El objetivo de este estudio es analizar las pautas de Fe i.v. que utilizamos en nuestra consulta de nefrologia.

\section{Pacientes y Métodos:}

Estudio prospectivo realizado en nuestra consulta externa de nefrología. Se recogen datos desde 2006 hasta el 1/abr/2013. Los contactos de las dosis ad- ministradas se registran desde abr/2008. El hierro administrado es hierro sacarosa. Inicialmente era Venofer ${ }^{\circledR}$ (Uriach) y después Feriv ${ }^{\circledR}$ (GES). La primera dosis se pone en 1 hora y el resto en 30-40 minutos, diluido en $40 \mathrm{ml}$ de suero fisiológico y administrada con bomba de perfusión (Alaris plus GH), tras canalizar una vena periférica con un abbocath $n^{\circ} 24 G$. Se registran las pautas de Fe i.v, la duración del mismo y los resultados obtenidos.

\section{Resultados:}

Se incluyen 172 pacientes, que tuvieron un total de 409 pautas. La mediana fue de 2 (IQ: 1 - 3). En algo más de la mitad de ellas $(n=211)$ la dosis administrada era de 2 ampollas. Respecto a la frecuencia osciló entre dosis única, (habitualmente asociado a dosis inicial) y cada 4 meses. La frecuencia más habitual fue cada mes, con un $44,3 \%$ de las pautas $(n=181)$. La mediana fue de 7,8 meses (IQ: $2,4-19,4$ ). Tenemos registradas un total de 1343 dosis administradas. El $62 \%$ de las dosis ha sido de 2 ampollas por dosis. La duración de la infusión osciló entre 30 y 60 minutos. La más habitual fue la de 40 minutos $(91,5 \%)$. No hemos tenido efectos adversos graves en ninguna de las administraciones realizadas. Actualmente 34 pacientes siguen administrándose hierro i.v. en nuestra consulta.

\section{Conclusiones:}

La administración de Fe i.v. por parte de la enfermera de la consulta de nefrología es factible, útil y segura. 


\section{Referencias Bibliográficas}

1. Administración de Hierro i.v. en la cta de nefrología Bibliografía: Locatelli F, Covix A, Eckardt KU,
Wiecek A Vanholder $\mathrm{R}$ on behalf of the ERA-EDTA ERBP Advisory Borrad. Anaemia management in patients with chronic kidney disease: a position statement by the Anaemia Working Group of European Renal Best Practice (ERBP). Nephrol Dial Transplant 2009; 24: 348-354. 